Research Article

\title{
Transient Reliability Evaluation Approach of Flexible Mechanism with GA-Extremum Neural Network
}

\author{
Yongjun Zhao, ${ }^{1}$ Cheng Lu, ${ }^{1}$ Chengwei Fei $D^{1},{ }^{1}$ Liqiang An, ${ }^{2}$ Yan Hu, ${ }^{3}$ Bo Huang, \\ and Liu Yuan ${ }^{3}$ \\ ${ }^{1}$ Department of Aeronautics and Astronautics, Fudan University, Shanghai 200433, China \\ ${ }^{2}$ Department of Mechanical Engineering, North China Electric Power University, Baoding 071003, China \\ ${ }^{3}$ Commercial Aircraft Engine Co., Ltd, AECC, Shanghai 200241, China
}

Correspondence should be addressed to Chengwei Fei; cwfei@fudan.edu.cn

Received 15 October 2020; Revised 20 October 2020; Accepted 30 October 2020; Published 16 November 2020

Academic Editor: Shun-Peng Zhu

Copyright ( 92020 Yongjun Zhao et al. This is an open access article distributed under the Creative Commons Attribution License, which permits unrestricted use, distribution, and reproduction in any medium, provided the original work is properly cited.

Efficient analytical model directly enhances the reliability evaluation of flexible mechanism under operation. In this paper, genetic algorithm-based extremum neural network (GA-ENN) is developed as reliability model by introducing the thoughts of extremum and genetic algorithm (GA) into artificial neural network to address the key problems comprising transient response and modeling precision in the dynamic reliability analysis of flexible mechanism in a time domain. The thought of extremum is adopted to simplify transient response process as one extremum value to the difficulty of dynamic reliability analysis induced by transient process response, and the GA is applied to find the optimal model parameters of reliability model. The dynamic reliability analysis of two-link flexible robot manipulator (TFRM) (a typical flexible mechanism) was implemented based on the GA-ENN method, regarding the input random variables of material density, elastic modulus, section sizes of components, and the output response of components' deformations. From the analysis, the comprehensive reliability of the TFRM is 0.951 when the allowable deformation is $1.8 \times 10^{-2} \mathrm{~m}$. Besides, the maximum deformations of the two components follow the normal distributions with the means of $1.45 \times 10^{-2} \mathrm{~m}$ and $1.69 \times 10^{-2} \mathrm{~m}$ and the standard variances of $6.77 \times 10^{-4} \mathrm{~m}$ and $4.08 \times 10^{-4} \mathrm{~m}$, respectively. Through the comparison of methods, it is illustrated that the developed GA-ENN improves the simulation efficiency and modeling accuracy by overcoming the problems of transient response and model parameter optimization in the dynamic reliability analysis of TFRM.

\section{Introduction}

As one of the important parts in mechanical system in robotics, satellite, aircraft, aeroengine, and so forth, flexible mechanism severely influences the safety and usability of mechanical system. Currently, the investigation of flexible mechanism attracts a lot of attention with the emphasis on the control strategy [1-3] and the modeling and solution of dynamic equation $[4,5]$. The reliability evaluation of flexible mechanism has become one interesting topic in ensuring the secure operation of mechanical system [6].

Numerous investigations on reliability analysis lead to the rapid development of reliability approaches. Response surface method (RSM, also called surrogate model) is widely applied in reliability analysis. Fei et al. developed decomposed-coordinated surrogate modeling strategy for compound function approximation and a turbine-blisk reliability evaluation [7], and the method was applied aeroengine blade-tip clearance and its components [8-10]; Li et al. employed support vector machine in structural reliability analysis [11]; Kaymaz applied Kriging method to complete structural reliability problems [12]; Xiong et al. presented a double weighted stochastic RSM for reliability analysis [13]; Gavin et al. gave the RSM-based high-order limit state functions for reliability analysis [14]; Ren et al. established neural network response surface model for reliability analysis based on artificial neural network (ANN) with high accuracy and nonlinear mapping capability [15]; 
Dai et al. applied this ANN-RSM to the regression analysis of complex structure limit state function to improve the accuracy of reliability analysis [16]. The above works mainly focus on structural reliability and prompt plentiful approaches on evaluating, analyzing, and designing structural reliability.

However, few efforts have been done on the reliability analysis of flexible mechanism. Flexible mechanism analysis involves nonlinearity, multicomponent coupling, and time variance (transient), so that it is inevitable that the reliability analysis of flexible mechanism is a complex simulation with large computational loads, strong coupling, and difficult calculation [17]. Due to the transient response and complicated analysis, the simulation efficiency and model precision of flexible mechanism reliability analysis are unacceptable if the response surface methods effectively used in structural reliability analysis are directly employed. To solve the issues, Song et al. studied the deformation response reliability analysis of flexible mechanism [6]. Zhang et al. completed the reliability evaluation and topology optimization of compliant mechanism by using level set method and first-order reliability method [18]. To improve the computational efficiency, Zhang et al. developed extremum RSM- (ERSM-) based quadratic polynomials for the reliability analysis of flexible manipulator by combining flexible multibody dynamics with modal comprehensive method and modal truncation technique [19]. Yang et al. proposed the backpropagation-ANN- (BP-ANN-) based Monte Carlo (MC) method for dynamic strength reliability analysis of flexible mechanism [20]. Reliability analytical model directly influences the precision and availability of flexible mechanism reliability evaluation under operation. Although the proposed ERSMs and BP-ANN model improve the efficiency and accuracy of flexible mechanism reliability analysis to some extent, the improved efficiency and precision are insufficient yet. This is because of the following: (1) as BP-ANN model is established, training algorithms have local optimization rather global optimization and difficult convergence; (2) the weights and thresholds (model parameters) in BP-ANN model are so imprecise that the BP-ANN model has low approximation accuracy; (3) modeling speed is too low to implement the reliability analysis of flexible mechanism due to noneffective transient processing.

Bayesian regularization algorithm (BRA) holds strong generalization ability and global optimization ability in searching for the model parameters by gradual approximation in training process [16]. Genetic algorithm (GA) effectively avoids premature convergence and local optimization in modeling process $[21,22]$. Therefore, it is important to adopt GA to improve the accuracy of BP-ANN model for flexible mechanism reliability analysis. The extremum thought in ERSM offers a measure to process the transient response problem in flexible mechanism reliability analysis.

The objective of this paper is to absorb the extremum thought and GA into ANN to develop a new approach, that is, GA-based extremum neural network (GA-ENN) method. This proposed method is adopted to improve the modeling precision and simulation efficiency in the reliability analysis of flexible mechanism by simplifying the response process as a response extremum value and employing GA to find the optimal parameters of ANN model, respectively. In what follows, Section 2 studies the GA-ENN method for the reliability analysis of flexible mechanism, including basic principle and GA-ENN model and GA. The basic thought of the reliability analysis of flexible mechanism based on the proposed GA-ENN is discussed in Section 3. The reliability analysis of TFRM is applied to validate the modeling accuracy and simulation efficiency of the proposed GA-ENN method in Section 4. In Section 5, the conclusions of this study are summarized.

\section{Theory and Method}

2.1. Artificial Neural Network. For the reliability analysis of flexible mechanism with quadratic polynomial-based response surface method, the quadratic polynomial is generally adopted as the fitted function to approximate to the limit state function of mechanism system. Due to the limitation of quadratic polynomials in approximating to the nonlinearity and function coupling, however, it is difficult to acquire the acceptable fitting accuracy when quadratic polynomials are directly applied to fit the limit state function of flexible mechanism [23]. To avoid the deficiency, this paper employs ANN instead of quadratic polynomials to fit the limit state function of flexible mechanism, because the ANN holds strong fitting ability and high fitting accuracy. The BP-ANN as a typical ANN holds the advantages of strong adaptability and the accurate fit ability to complex function [24]. When $y$ and $x=\left(x_{1}, x_{1}, \ldots, x_{m}\right)$ are output response and input variable, respectively, in respect of ANN model, the function relationship between output response $y$ and input variables $x_{i}$ is expressed as

$$
y=f_{2}\left(\sum_{j=1}^{n} \omega_{j k} f_{1}\left(\sum_{i=1}^{m} \omega_{i j} x_{i}+b_{j}\right)+b_{k}\right),
$$

where $\omega_{j k}$ is the connection weight between the $j$ th node of input layer and the $k$ th node of hidden layer; $b_{j}$ is the $j$ th threshold value of hidden layer; $\omega_{i j}$ is the connection weight between the $i$ th node of hidden layer and the $j$ th node of output layer; $b_{k}$ is the $k$ th threshold value of output layer; $f_{1}(\cdot)$ is the transfer function between input layer and hidden layer; $f_{2}(\cdot)$ is the transfer function between hidden layer and output layer; $m$ and $n$ are the numbers of nodes in input layer and hidden layer, respectively.

Training algorithm is one of the key techniques of establishing the BP-ANN model. This paper adopts Bayesian regularization algorithm (BRA) as the training algorithm of ANN because the algorithm holds strong generalization ability, global optimization capacity, and rapid convergence ability. The BRA is promising to improve the generalization capacity of ANN model by avoiding overfitting problem and finding the optimized weights and threshold values in training process [16]. The BRA-based training performance function $\boldsymbol{E}$ of is

$$
e=\alpha_{1} e_{e}+\alpha_{2} e_{\omega}
$$

We have 


$$
\left\{\begin{array}{l}
\boldsymbol{e}_{e}=\frac{1}{2}\left\|\varepsilon\left(\boldsymbol{v}^{k}+Z\left(\boldsymbol{v}^{k+1}-\boldsymbol{v}^{k}\right)\right)\right\|^{2}+\lambda\left\|\boldsymbol{v}^{k+1}-\boldsymbol{v}^{k}\right\|^{2}, \\
e_{\omega}=\frac{1}{m} \sum_{j=1}^{m} \omega_{j}^{2},
\end{array}\right.
$$

where $e$ is the BRA function; $e_{e}$ and $e_{\omega}$ are the mean sum of squares of the network error and the sum of squares of network weights; $\alpha_{1}$ and $\alpha_{2}$ are the proportional coefficients of $e_{e}$ and $e_{\omega}$, respectively; $w_{j}$ is the weight of $\mathrm{ANN} ; \varepsilon$ is the expected error function of output response; $\boldsymbol{v}$ is the vector of weight and threshold values for network layers; $k$ is the $k$ th iteration; $Z$ is the Jacobian matrix of $\varepsilon ; \lambda$ is the iteration variable.

2.2. Genetic Algorithm. Relative to quadratic polynomialbased response surface method, ANN model has the potential of reducing computational loads and enhancing simulating efficiency. However, the traditional ANN model has local optimization and low approximation accuracy so that the network generalization ability is deficient. Intelligent operator may effectively avoid the problems of premature convergence and local optimization in training ANN by searching for the optimal initial weight and threshold. Therefore, it is important to design the intelligent operator to improve the accuracy of ANN model.

As one typical intelligent operator, genetic algorithm (GA) can automatically determine searching space and adaptively adjust searching direction without the restriction of differentiable or continuous objective function. Meanwhile, the GA has the abilities of parallel processing and strong robustness [21] and effectively approximates to optimal weight and threshold. In this paper, the GA is adopted to find the optimal weights and threshold to improve the modeling accuracy of ANN [21, 25]. In the GA, the initial population includes network weights and thresholds, and each individual in the population contains all genetic information. Because the network training error is the adaptive value of the individual, the smaller the individual adaptive value is, the better the individual is. Genetic operator includes three operators: selection, cross, and mutation. The design details of the three operators are summarized as follows.

Based on the adaptive proportional roulette wheel method [26], the selected probability of each individual is

$$
P_{i}=\frac{1}{\sum_{j=1}^{N}\left(f_{i} / f_{j}\right)},
$$

where $P_{i}$ represents the probability of the $i$ th individual; $f_{i}$ is the adaptive value of the $i$ th individual; $f_{j}$ is the fitness value of the $j$ th individual; $N$ is the number of individuals in population.

Cross operation is commonly finished by the real number cross method [27]. The cross operation of the $k$ th chromosome $a_{k}$ and $l$ th chromosome $a_{l}$ on $j$ th point is

$$
\left\{\begin{array}{l}
a_{i j}^{(k+1)}=a_{i j}^{(k)}(1-r)+a_{l j}^{(k)} r, \\
a_{l j}^{(k+1)}=a_{l j}^{(k)}(1-r)+a_{i j}^{(k)} r .
\end{array}\right.
$$

where $k$ is the $k$ th iteration; $a_{i j}$ is the $j$ th bit of the $i$ th chromosome; $a_{l j}$ is the $j$ th bit of the $l$ th chromosome; $r$ is the random number in $[0,1]$.

The mutation of the $j$ th gene of the $i$ th individual is denoted by

$$
a_{i j}^{(k+1)}= \begin{cases}a_{i j}^{(k)}+\left(a_{i j}^{(k)}-a_{\max }\right) \times t\left(1-\frac{k}{K}\right), & r>0.5, \\ a_{i j}^{(k)}+\left(a_{\min }-a_{i j}^{(k)}\right) \times t\left(1-\frac{k}{K}\right), & r \leq 0.5,\end{cases}
$$

where $a_{\max }$ and $a_{\min }$ indicate the upper bound and bottom bound of gene $a_{i j}$, respectively; $K$ is the maximum number of iterations.

2.3. Genetic Algorithm-Based Extremum Neural Network $(G A-E N N)$. In this section, we develop the GA-ENN method in the foundation of GA and ANN introduced from the extremum thought. The architecture of GA-ENN is shown in Figure 1.

As shown in Figure 1, the basic thought of GA-ENN method is summarized as follows:

(i) The limit state equation of flexible mechanism system is fitted by fitting extremum response surface models (i.e., GA-ENN model) to the input parameter $x_{j}$ and extremum output $Y_{j}\left(t, x_{j}\right)$.

(ii) The established GA-ENN model of this system is analyzed in the time domain $[0, \mathrm{~T}]$ replacing the nonlinear dynamic differential equations to gain dynamic extremum output response $Y_{j \max }\left(x_{j}\right)$.

From the above analysis, the GA-ENN transforms the stochastic response process in the dynamic reliability analysis of flexible mechanism into one random extremum response in the time domain in each calculation. Corresponding to the sample set $x$ of random variables, all extremum responses are employed to fit the GA-ENN model. In the dynamic reliability analysis with the GA-ENN method, only the extremum of output response in time domain is calculated rather than all output responses in the time domain. Therefore, the proposed approach (GA-ENN) can potentially reduce simulation time and improve analytical efficiency.

\section{Procedure of Flexible Mechanism Reliability Analysis with GA-ENN}

To solve the limitations of the traditional response surface method in the reliability analysis of flexible mechanism, the developed GA-ENN is to enhance the nonlinear searching ability of intelligent operator and the nonlinear mapping ability of ANN, reduce the computational load, and improve computational efficiency and computational accuracy in the reliability analysis of flexible mechanism. The reliability analysis procedure of flexible mechanism with GA-ENN is shown in Figure 2. 


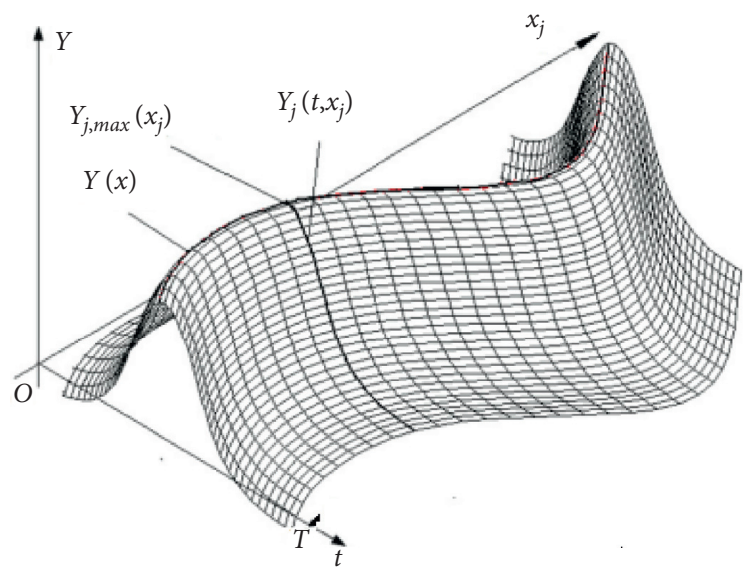

FIGURE 1: The architecture of GA-ENN method. $x$ is the set of input samples; $x_{j}$ is the $j$ th input sample in the set of input samples $x ; Y_{j}\left(t, x_{j}\right)$ is the output response process corresponding to $x_{j}$ in time domain $[0, T] ; Y_{j \max }\left(x_{j}\right)$ is the maximum value of $Y_{j}\left(t, x_{j}\right)$ in time domain $[0, \mathrm{~T}]$; $Y(x)$ is extremum response surface model (ANN model) built by the set of input samples $x$.

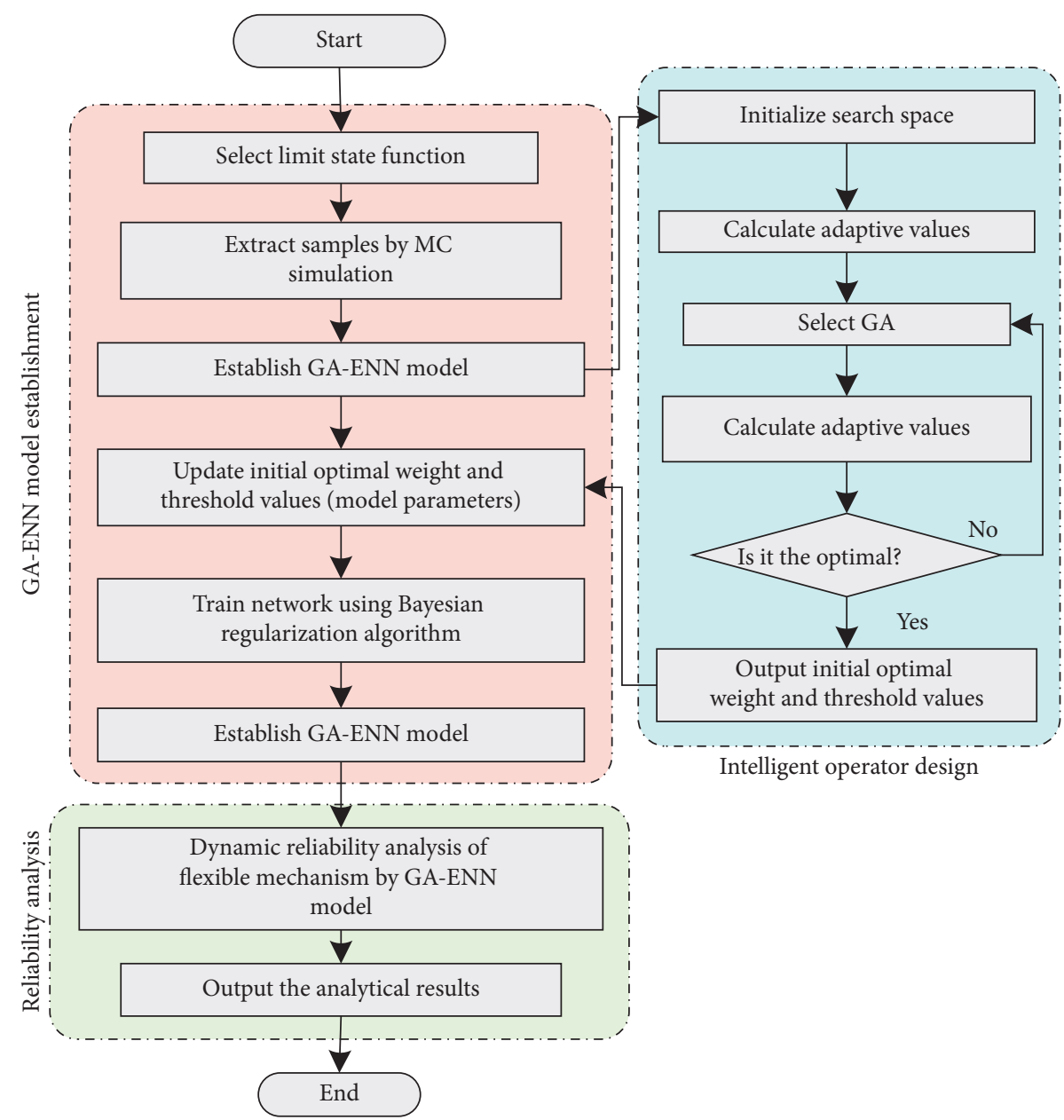

FIGURE 2: Reliability analysis procedure of flexible mechanism based on BRA/GA-ENN. 
As shown in Figure 2, the reliability analysis procedure of flexible mechanism based on GA-ENN can be illustrated as follows:

(i) Step 1: Establish the limit state function of flexible mechanism by combining the comprehensive modal theory and multibody dynamics theory.

(ii) Step 2: Build ANN model in respect of the node number of each layer of ANN and enough training samples by extracting limit state function using Latin hypercube sampling method [28].

(iii) Step 3: Initialize search space and calculate individual adaptive values and find the optimal solution of intelligent search according to individual adaptive values.

(iv) Step 4: Judge whether the optimal individual in the updated population satisfies the requirement of design. If no, return to intelligent operator (GA) to continually execute until meeting the requirement of design. If yes, continue to Step 5.

(v) Step 5: Return to main program after gaining the optimal weight and threshold values.

(vi) Step 6: Apply BRA to establish ANN model, that is, GA-ENN model.

(vii) Step 7: Complete the reliability analysis of flexible mechanism based on the established GA-ENN model and output the results.

\section{The Reliability Analysis of Flexible Mechanism}

4.1. Formulation of Problems. In this paper, a TFRM is regarded as the object of study on the reliability analysis of flexible mechanism based on GA-ENN. The simplified model of TFRM is shown in Figure 3. In this TFRM, the components (or subcomponents) of the manipulator are considered as a homogeneous Euler beam. Mass loads enforce at the joint between component-1 and component-2. The end of arm is assumed to be concentrated masses with ignoring rotational inertia and damping of rotor motor. For two manipulators, the lengths are $l_{1}$ and $l_{2}$; the masses are $m_{1}$ and $m_{2}$; the driving torques are $\tau_{1}(t)$ and $\tau_{2}(t)$, respectively. To analyze the motion condition of the two manipulators (components), $x_{1}-y_{1}$ local coordinate is built for component-1, and $x_{2}-y_{2}$ local coordinate is established for component-2. $y_{1}$ and $y_{2}$ represent the elastic deformations of component- 1 and component-2, respectively. $t$ is movement time. The movements of two moving local coordinates are described by the azimuths $\theta_{1}(t)$ and $\theta_{2}(t)$, respectively.

Based on the integrated mode method [19], the elastic deformations of the two components in the corresponding local coordinate systems are analyzed. The shape functions ( $\varphi_{1}$ for number-1 and $\varphi_{2}$ for number-2) of the two components are

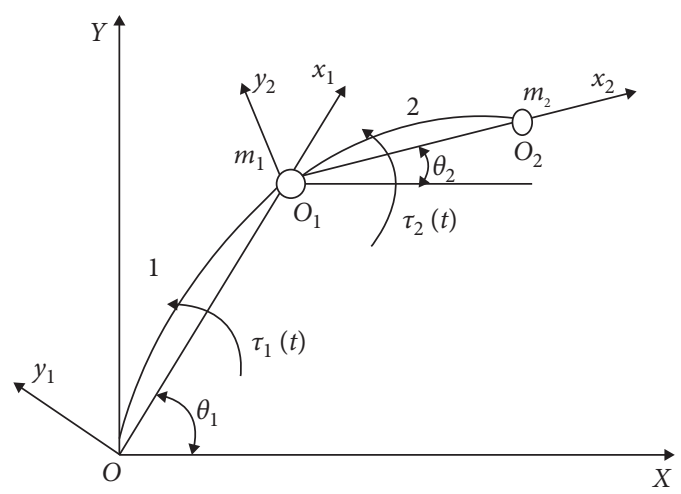

Figure 3: Two-link flexible robot manipulator model.

$$
\left\{\begin{array}{l}
\varphi_{1}(x)=\sin \left(\frac{\pi x}{l}\right), \\
\varphi_{2}(x)=\sin \left(\frac{2 \pi x}{l}\right) .
\end{array}\right.
$$

The elastic deformations of components change with time. The elastic deformations $y_{1}\left(t, x_{1}\right)$ and $y_{2}\left(t, x_{2}\right)$ for component- 1 and component- 2 on $y$ direction are indicated, respectively, by

$$
\left\{\begin{array}{l}
y_{1}\left(t, x_{1}\right)=\sum_{i=1}^{n} g_{i}(\mathrm{t}) \varphi_{i}\left(x_{1}\right), \\
y_{2}\left(t, x_{2}\right)=\sum_{i=1}^{n} u_{i}(\mathrm{t}) \varphi_{i}\left(x_{2}\right) .
\end{array}\right.
$$

The generalized coordinate $q(t)$ is $q(t)=\left[q_{1}, q_{2}, q_{3}, q_{4}, q_{5}, q_{6}\right]^{T}=\left[\theta_{1}(t), g_{1}(t), g_{2}(t), \theta_{2}(t), u_{1}(t), u_{2}(t)\right]^{T}$,

where $g_{i}(t)$ is the $i$ th-order elastic coordinate of component$1 ; u_{i}(t)$ is the $i$ th-order elastic coordinate of component- 2 .

According to Lagrange equations, the dynamics equation of the TFRM is

$$
Q_{k}=M \ddot{q}+\dot{M} \dot{q}-\frac{\partial}{\partial q}\left(\frac{1}{2} \dot{q}^{T} M \dot{q}\right)+K q+\frac{\partial U_{g}}{\partial q} .
$$

where $U_{g}$ is gravitational potential energy; $M$ is mass matrix; $K$ is stiffness matrix; $Q_{k}(t)$ is total force corresponding to the moment of rotation computed by the virtual work method [19].

The main failure mode of TFRM is deformation failure, so that the deformation reliability analysis is concerned in this study [19].

4.2. Selection of Random Variables. The basic parameters of TFRM are mass $M$, length $L$, and driving torque $\tau$, which are shown in Table 1 . The density $\rho$, elastic modulus $E$, and section size $h$ and $b$ are the random parameters in the reliability analysis of TFRM as shown in Table 2, in which the variables follow normal distributions and are independent mutually. 
TABLE 1: Basic parameters of component-1 and component-2.

\begin{tabular}{lccc}
\hline Parameters & Mass $M(\mathrm{~kg})$ & Length $L(\mathrm{~m})$ & Drive torque $\tau(\mathrm{N} \cdot \mathrm{m})$ \\
\hline Component-1 & 5.5 & 0.75 & $215 \sin ^{3}(2 \pi t)-62$ \\
Component-2 & 7.5 & 0.75 & $75 \sin ^{3}(2 \pi t)+15$ \\
\hline
\end{tabular}

4.3. GA-ENN Modeling. Within the variance range of random variables, 100 groups of input data are extracted by the MC method. The output responses (maximum deformation) of component-1 and component-2 are calculated by limit state function. The 100 groups of data are normalized and used as the network training samples. According to equation (11), the network hidden lawyer neuron is denoted as $k_{i}=2 \sim 9(i=1,2)$. Through the comparison of the network training error, the number of hidden layer nodes is selected, that is, $k_{1}=k_{2}=3$, as shown in Table 3.

$$
n=\sqrt{n_{i}+n_{o}}+a
$$

where $n$ is the number of hidden layers; $n_{i}$ is the number of input nodes; $n_{o}$ is the number of output nodes; $a$ is the arbitrary constant in $[16,17]$.

The 4-3-1 three-layer network structure was chosen as the GA-ENN model, in which the transfer functions from input layer to hidden layer and from hidden layer to output layer and the training function are "tansig," "purelin," and "trainbr," respectively. Particle dimension $\mathbf{v}=19$ and particle number $N=40$ were selected. Through 100 iterations, the optimal adaptive value curves are shown in Figure 4.

The initial optimal weights and threshold values are inputted into GA-ENN model. After the network training with the BRA, the function of GA-ENN is obtained, in which the weight and threshold levels of two components are shown in the two following equations:

$$
\begin{aligned}
& \left\{\begin{array}{l}
w_{1}=\left[\begin{array}{llll}
1.5369 & 1.5369 & 1.5369 & 1.5369 \\
7.6994 & 7.6994 & 7.6994 & 7.6994 \\
0.8209 & 0.8209 & 0.8209 & 0.8209
\end{array}\right], \\
b_{1}=\left[\begin{array}{l}
-1.4668 \\
2.6843 \\
3.9841
\end{array}\right] \\
w_{2}=\left[\begin{array}{lll}
-0.2787 & 1.1137 & 0.2567
\end{array}\right] \\
b_{2}=\left[\begin{array}{lll}
0.8563
\end{array}\right]
\end{array}\right. \\
& \left\{\begin{array}{l}
w_{1}=\left[\begin{array}{cccc}
-5.6841 & -5.6841 & -5.6841 & -5.6841 \\
0.1892 & 0.1892 & 0.1892 & 0.1892 \\
-0.3698 & -0.3698 & -0.3698 & -0.3698
\end{array}\right], \\
b_{1}=\left[\begin{array}{c}
0.6982 \\
-3.65781 \\
-0.8129
\end{array}\right], \\
w_{2}=\left[\begin{array}{lll}
-23831 & -3.9841 & 0.9218
\end{array}\right], \\
b_{2}=[-1.9853] .
\end{array}\right.
\end{aligned}
$$

4.4. Reliability Analysis. Through 10,000 simulations on the GA-ENN by the MC simulation, the output responses are obtained by inversed normalization. The deformation curve, deformation distribution, and deformation cumulative function of two components are shown in Figures 5-7. Assuming that the allowable deformation is $1.8 \times 10^{-2} \mathrm{~m}$, the results of reliability analysis are listed in Table 4 .

As shown in Figures 5-7, the maximum dynamical deformation values of two components are evenly distributed around the mean values and approximately obey a normal distribution with the mean values of $1.45 \times 10^{-2} \mathrm{~m}$ and $1.69 \times 10^{-2} \mathrm{~m}$ and the standard deviations of $6.77 \times 10^{-4} \mathrm{~m}$ and $4.08 \times 10^{-4} \mathrm{~m}$, respectively.

As revealed by Table 4, we obtain the reliability degrees 1 and 0.951 for component- 1 and component-2, respectively. TFRM is a continuous system. The system stiffness reliability is equal to the product of the reliability degrees of the two components [16]. Thus, the reliability degree of TFRM system is $P_{y}=P_{y 1} \cdot P_{y 2}=0.951$. Through 1000 simulations on the BPANN models for two components, the computational times of two components are $0.126 \mathrm{~s}$ and $0.158 \mathrm{~s}$, respectively.

4.5. Method Validation. To validate the GA-ENN, the dynamic reliability analyses of TFRM were conducted with MC simulation (direct method), extremum response surface method (denoted by ERSM) [29], and the proposed GAENN under the same computational conditions. All analyses are performed by an Intel (R) Core (TM) i7-9700T desktop computers with $2.00 \mathrm{GHz}$ CPU and 16 GB RAM. The ERSM is developed by introducing the thought of extremum into quadratic polynomial-based response surface method. The simulation time and reliability precision are shown in $\mathrm{Ta}-$ ble 5 and Figure 8 as well as Table 6 and Figure 9 .

As revealed in Table 5 and Figure 8, ERSM and GA-ENN are much better than the MC method in computation time, indicating that surrogate model approaches hold higher simulation speed and analytical efficiency than Direct FE model analysis for the same number of simulations. With the increase of simulation times, the computational efficiency of GA-ENN is higher than ERSM, indicating that GA-ENN method holds higher simulation speed than the ERSM, because the GA-based parameter optimization for GA-ENN approach is faster than the least square method for ERSM in acquiring the model parameters. Therefore, the proposed GA-ENN achieves higher efficiency than the quadratic polynomial-based ERSM.

As illustrated in Table 6 and Figure 9, the GA-ENN is almost consistent with MC method in the calculation accuracy, which is higher than the calculation accuracy of ERSM. This is because adopting GA can gain more appreciated model parameters, while the least square method is 
TABLE 2: Random parameters of component-1 and component-2.

\begin{tabular}{lcccccc}
\hline Variables & Density $\rho\left(\mathrm{kg} \cdot \mathrm{m}^{-3}\right)$ & Elasticity modulus $E(\mathrm{~Pa})$ & \multicolumn{2}{c}{ Component-1 } & \multicolumn{2}{c}{ Component-2 } \\
& & & $h_{1}(\mathrm{~m})$ & $b_{1}(\mathrm{~m})$ & $h_{2}(\mathrm{~m})$ & $b_{2}(\mathrm{~m})$ \\
\hline Mean & 2067 & $4.0875 \times 10^{9}$ & 0.06 & 0.015 & 0.04 & 0.01 \\
Standard deviation & 10 & $2.0438 \times 10^{8}$ & 0.04 & 0.01 & 0.0267 & 0.0067 \\
\hline
\end{tabular}

TABLE 3: Network training error with different hidden neuron number.

\begin{tabular}{lccccccc}
\hline Neuron number & 2 & 3 & 4 & 5 & 6 & 7 & 8 \\
\hline Network-1 error & 0.12 & 0.10 & 0.13 & 0.13 & 0.12 & 0.20 & 0.22 \\
Network-2 error & 0.17 & 0.16 & 0.16 & 0.17 & 0.19 & 0.18 & 0.20 \\
\hline
\end{tabular}

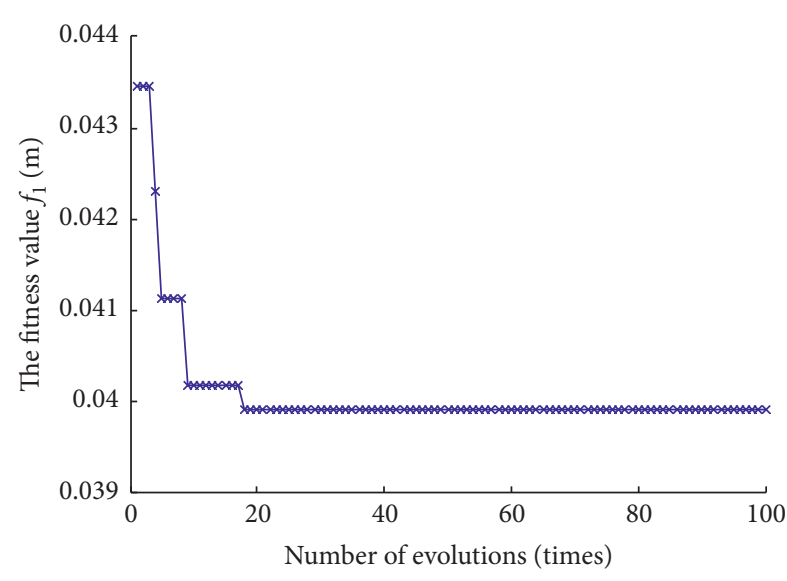

(a)



(b)

Figure 4: Optimal fitness value curves. (a) Component-1. (b) Component-2.

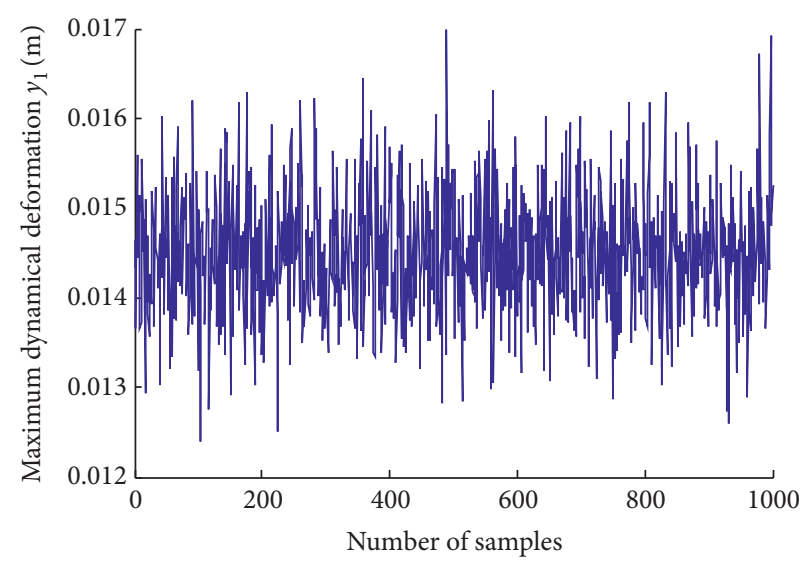

(a)

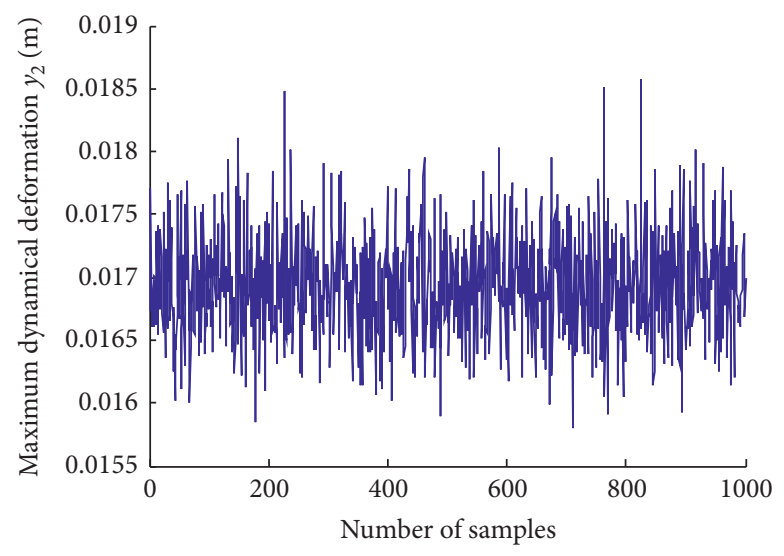

(b)

Figure 5: Maximum deformations of midpoint on the two components. (a) Component-1. (b) Component-2.

unworkable in acquiring the optimal modeling parameters. It is demonstrated that the GA-ENN holds higher accuracy than ERSM [19, 29-31]. The GA-ENN greatly save computational time and improved calculation efficiency while keeping computational accuracy. Therefore, the proposed GA-ENN is verified to be a feasible and effective 


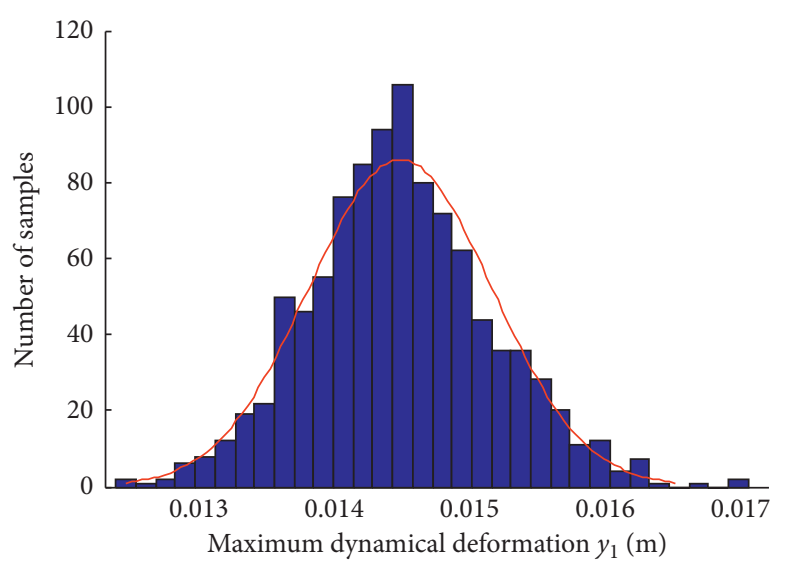

(a)



(b)

Figure 6: Maximum deformation distributions of two components. (a) Component-1. (b) Component-2.

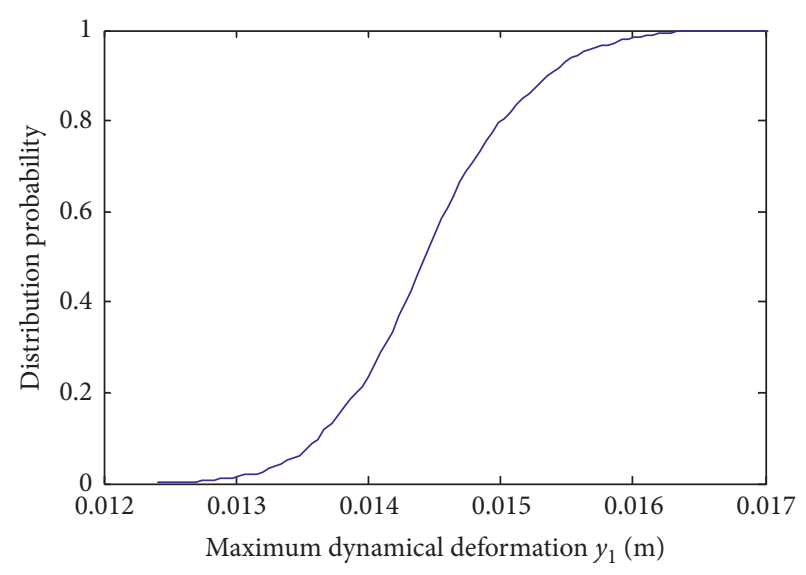

(a)

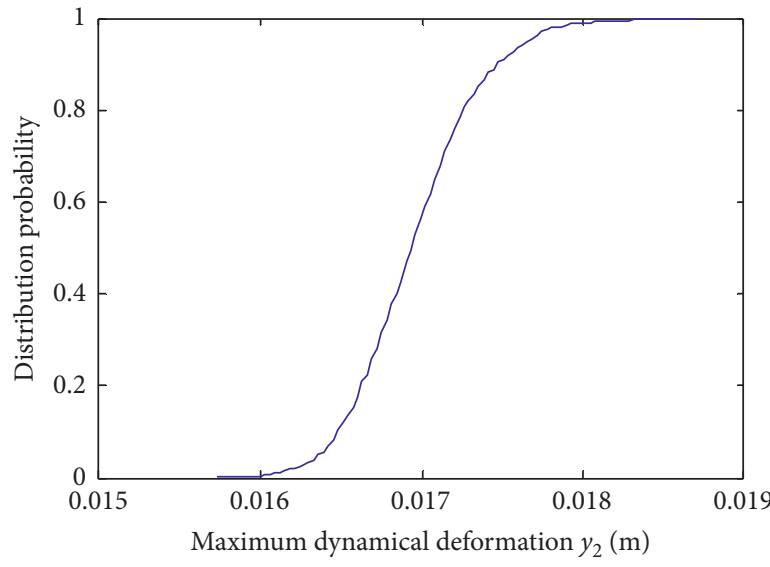

(b)

Figure 7: The cumulative curves of TFRM deformations. (a) Component-1. (b) Component-2.

TABLE 4: Analytical results of TFRM deformation reliability.

\begin{tabular}{lcccccc}
\hline Components & Failure number & Reliability & Mean $\left(\times 10^{-3} \mathrm{~m}\right)$ & Standard deviation $\left(\times 10^{-3} \mathrm{~m}\right)$ & Distribution & Computational time $(\mathrm{s})$ \\
\hline Component-1 & 0 & 1 & 14.5 & 0.677 & Normal & 0.126 \\
Component-2 & 49 & 0.951 & 16.9 & 0.408 & Normal & 0.158 \\
\hline
\end{tabular}

TABLE 5: Simulation time of TFRM reliability analyses

\begin{tabular}{|c|c|c|c|c|}
\hline \multirow{2}{*}{ Methods } & \multicolumn{4}{|c|}{ Simulation time under different simulations (s) } \\
\hline & $10^{2}$ & $10^{3}$ & $10^{4}$ & $10^{5}$ \\
\hline MC method & $2.94 \times 10^{3}$ & $2.98 \times 10^{4}$ & $2.99 \times 10^{5}$ & - \\
\hline ERSM & 0.36 & 0.59 & 1.68 & 125.28 \\
\hline GA-ENN & 0.14 & 0.25 & 0.49 & 36.08 \\
\hline
\end{tabular}




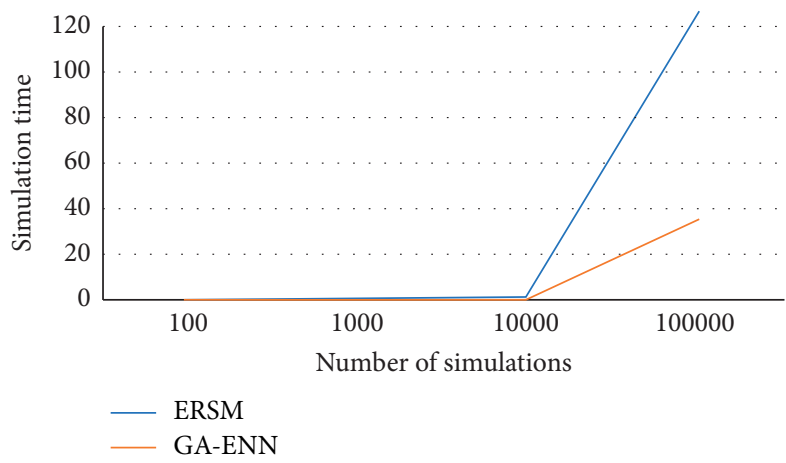

Figure 8: Computing time of ERSM and GA-ENN methods.

TABLE 6: Evaluation precision of TFRM reliability analyses

\begin{tabular}{|c|c|c|c|c|c|}
\hline \multirow{2}{*}{ Number of samples } & \multicolumn{3}{|c|}{ Reliability degree } & \multicolumn{2}{|c|}{ Precision (\%) } \\
\hline & MC method & ERSM & GA-ENN & ERSM & GA-ENN \\
\hline $10^{2}$ & 0.9700 & 0.9800 & 0.9700 & 99 & 99 \\
\hline $10^{3}$ & 0.9520 & 0.9610 & 0.9510 & 99.1 & 99.9 \\
\hline $10^{4}$ & 0.9865 & 0.9538 & 0.9866 & 96.7 & 99.99 \\
\hline $10^{5}$ & - & 0.9568 & 0.9861 & - & - \\
\hline
\end{tabular}

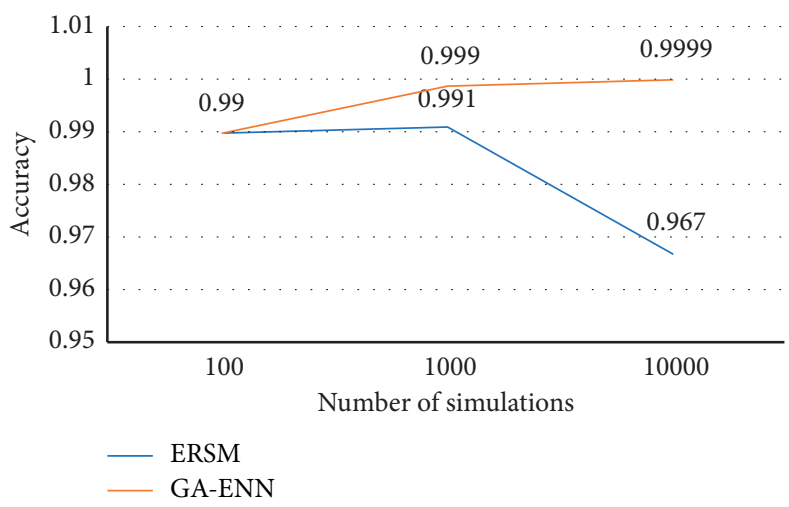

FIGURE 9: Precision of ERSM and GA-ENN methods.

method in dynamic reliability analysis of flexible mechanism.

\section{Conclusions}

(1) A genetic algorithm- (GA-) based extremum neural network (GA-ENN) method is proposed by combining artificial neural network (ANN), genetic algorithm (GA), and extremum thought to improve the modeling precision and simulation efficiency in the reliability analysis of flexible mechanism.

(2) The total reliability degree of TFRM is 0.951 and the reliability degrees of component- 1 and component- 2 are 1 and 0.951 , respectively. Meanwhile, the maximum deformations of component- 1 and component- 2 follow the normal distribution with the means of $1.45 \times 10^{-2} \mathrm{~m}$ and $1.69 \times 10^{-2} \mathrm{~m}$ and the standard variances of $6.77 \times 10^{-4} \mathrm{~m}$ and $4.08 \times 10^{-4} \mathrm{~m}$ as well.
(3) The developed GA-ENN holds high modeling precision and simulation efficiency in respect of the comparison of methods.

(4) The advantages of GA-ENN are more obvious with the increase of simulation number, which supports that the GA-ENN model has higher computational efficiency and precision and better robustness than the quadratic polynomial function in dynamic reliability analysis.

(5) The efforts of this study provide a promising way for the reliability analysis and optimization of flexible mechanism and complex structures with the emphasis on high-precise model and high-simulation speed.

\section{Data Availability}

The data used to support the findings of this study are included within the article. 


\section{Conflicts of Interest}

The authors declare that there are no conflicts of interest regarding the publication of this article.

\section{Acknowledgments}

This paper is cosupported by the National Natural Science Foundation of China (Grants nos. 51975124 and 51675179), Research Start-up Fund of Fudan University (Grant no. FDU38341), and Shanghai International Cooperation Project of One Belt and One Road of China (Grant no. 20110741700). The authors would like to acknowledge them.

\section{References}

[1] X. Zhang, W. Xu, and S. S. Nair, "Comparison of some modeling and control issues for a flexible two link manipulator," ISA Transactions, vol. 43, no. 4, pp. 509-525, 2004.

[2] Z. Shun-Peng, K. Behrooz, C. Subrata, and T. Nguyen-Thoi, "Novel probabilistic model for searching most probable point in structural reliability analysis," Computer Methods in Applied Mechanics and Engineering, vol. 366, 2020.

[3] A. Green and J. Z. Sasiadek, "Dynamics and trajectory tracking control of a two-link robot manipulator," Journal of Vibration and Control, vol. 10, no. 10, pp. 1415-1440, 2004.

[4] X. Zhang, "Integrated optimal design of flexible mechanism and vibration control," International Journal of Mechanical Sciences, vol. 46, no. 11, pp. 1607-1620, 2004.

[5] T. V. Zavrazhinam, "Dynamics of robot manipulator with elastically flexible links and drive mechanisms," Journal of Automation and Information Sciences, vol. 37, no. 1, pp. 5565, 2005.

[6] L.-K. Song, G.-C. Bai, C.-W. Fei, and R. P. Liem, “Transient probabilistic design of flexible multibody system using a dynamic fuzzy neural network method with distributed collaborative strategy," Proceedings of the Institution of $\mathrm{Me}$ chanical Engineers, Part G: Journal of Aerospace Engineering, vol. 233, no. 11, pp. 4077-4090, 2019.

[7] C. W. Fei, C. Lu, and R. P. Liem, "Decomposed-coordinated surrogate modelling strategy for compound function approximation and a turbine blisk reliability evaluation," Aerospace Science and Technology, vol. 95, 2019.

[8] C.-W. Fei and G.-C. Bai, "Distributed collaborative probabilistic design for turbine blade-tip radial running clearance using support vector machine of regression," Mechanical Systems and Signal Processing, vol. 49, no. 1-2, pp. 196-208, 2014.

[9] C.-W. Fei, H. Li, H.-T. Liu, C. Lu, B. Keshtegar, and L.-Q. An, "Multilevel nested reliability-based design optimization with hybrid intelligent regression for operating assembly relationship," Aerospace Science and Technology, vol. 103, 2020.

[10] C. Fei, H. Liu, Z. Zhu, L. An, S. Li, and C. Lu, "Whole-process design and experimental validation of landing gear lower drag stay with global/local linked driven optimization strategy," Chinese Journal of Aeronautics, 2020.

[11] H.-S. Li, Z.-Z. Lü, and Z.-F. Yue, "Support vector machine for structural reliability analysis," Applied Mathematics and Mechanics, vol. 27, no. 10, pp. 1295-1303, 2006.

[12] I. Kaymaz, "Application of Kriging method to structural reliability problems," Structural Safety, vol. 27, no. 2, pp. 133-151, 2005.
[13] F. Xiong, Y. Liu, Y. Xiong, and S. Yang, "A double weighted stochastic response surface method for reliability analysis," Journal of Mechanical Science and Technology, vol. 26, no. 8, pp. 2573-2580, 2012.

[14] H. P. Gavin and S. C. Yau, "High-order limit state functions in the response surface method for structural reliability analysis," Structural Safety, vol. 30, no. 2, pp. 162-179, 2008.

[15] Y. Ren and G. Bai, "New neural network response surface methods for reliability analysis," Chinese Journal of Aeronautics, vol. 24, no. 1, pp. 25-31, 2011.

[16] H. Z. Dai, W. Zhao, W. Wang, and Z. G. Cao, “An improved radial basis function network for structural reliability analysis," Journal of Mechanical Science and Technology, vol. 25, no. 9, pp. 2151-2159, 2011.

[17] Y. B. Han, G. C. Bai, and X. Y. Li, "Dynamic reliability analysis of flexible mechanism based on support vector machine," Journal of Mechanical Engineering, vol. 11, no. 5, pp. 86-92, 2014.

[18] X. Zhang and G. Ouyang, "A level set method for reliabilitybased topology optimization of compliant mechanisms," Science in China Series E: Technological Sciences, vol. 51, no. 4, pp. 443-455, 2008.

[19] C.-Y. Zhang and G.-C. Bai, "Extremum response surface method of reliability analysis on two-link flexible robot manipulator," Journal of Central South University, vol. 19, no. 1, pp. 101-107, 2012.

[20] X. Yang, R. Yuan, and L. Wang, "Dynamical strength reliability analysis of a flexible mechanism using neural networks," Mechanical Science and Technology for Aerospace Engineering, vol. 27, no. 4, pp. 462-465, 2008.

[21] F. Dai, N. Kushida, L. Shang, and M. Sugisaka, "A survey of genetic algorithm-based face recognition," Artificial Life and Robotics, vol. 16, no. 2, pp. 271-274, 2011.

[22] X.-P. Niu, R.-Z. Wang, D. Liao, S.-P. Zhu, X.-C. Zhang, and B. Keshtegar, "Probabilistic modeling of uncertainties in fatigue reliability analysis of turbine bladed disks," International Journal of Fatigue, vol. 142, p. 105912, 2020.

[23] C. Lu, Y.-W. Feng, R. P. Liem, and C.-W. Fei, "Improved kriging with extremum response surface method for structural dynamic reliability and sensitivity analyses," Aerospace Science and Technology, vol. 76, pp. 164-175, 2018.

[24] C.-Y. Zhang, L.-K. Song, C.-W. Fei, G.-P. Hao, and L.-J. Liu, "Reliability-based design optimization for flexible mechanism with particle swarm optimization and advanced extremum response surface method," Journal of Central South University, vol. 23, no. 8, pp. 2001-2007, 2016.

[25] C. Lu, C.-W. Fei, H.-T. Liu, H. Li, and L.-Q. An, "Moving extremum surrogate modeling strategy for dynamic reliability estimation of turbine blisk with multi-physics fields," Aerospace Science and Technology, vol. 106, 2020.

[26] S. S. Venkatesh and D. Mishra, "Variable search space converging genetic algorithm for solving system of non-linear equations," Journal of Intelligent Systems, vol. 30, no. 1, pp. 142-164, 2020.

[27] L.-K. Song, C.-W. Fei, G.-C. Bai, and L.-C. Yu, "Dynamic neural network method-based improved PSO and BR algorithms for transient probabilistic analysis of flexible mechanism," Advanced Engineering Informatics, vol. 33, pp. 144-153, 2017.

[28] C. Lu, Y.-W. Feng, C.-W. Fei, and S.-Q. Bu, "Improved decomposed-coordinated kriging modeling strategy for dynamic probabilistic analysis of multicomponent structures," IEEE Transactions on Reliability, vol. 69, no. 2, pp. 440-457, 2020. 
[29] C. W. Fei, G. C. Bai, and C. Tian, "Extremum response surface method for casing radial deformation probabilistic analysis," Journal of Aerospace Information System, vol. 10, no. 1, pp. 47-52, 2013.

[30] Z. Shun-Peng, K. Behrooz, B. Mansour, H. Peng, and T. Nguyen-Thoi, "Novel hybrid robust method for uncertain reliability analysis using finite conjugate map," Computer Methods in Applied Mechanics and Engineering, vol. 371, 2020.

[31] D. Liao, S.-P. Zhu, B. Keshtegar, G. Qian, and Q. Wang, "Probabilistic framework for fatigue life assessment of notched components under size effects," International Journal of Mechanical Sciences, vol. 181, 2020. 